\title{
Aspects of the Schwinger Variational Principle Formulation
}

J.L.S. LINO, A.R. de FARIAS, N.S. ARAÚJO, Núcleo de Pesquisas em Matemática e Matemática Aplicada, Universidade Braz Cubas UBC, Campus I, 0877-380 Mogi das Cruzes, SP, Brazil

\begin{abstract}
The application of the Schwinger variational principle-SVP and, in particular,the second Born approximation to the evaluation of scattering amplitude and integral cross sections for eletron-atom impact is discussed. The SVP involving the expansion of the wave function as a plane wave is shown to be an effective approach and several details of the method are discussed.
\end{abstract}

\section{Introduction}

Investigation and development of alternative variational procedures are of great importance in dealing with scattering problems [1]. On one hand, the variational procedures are more effective than the direct numerical methods which, when applied to realistic problems, can tax even the largest computers. On the other hand, there is still a clear need for workers to determine the relative advantages of alternative variational methods in scattering theory [1]. Several recent applications have shown that the Scwhinger variational principle (SVP) is an effective and promising approach to the electron - atom (or molecule) collision problem. The Schwinger method, which allows one to expand trial scattering functions exclusively in terms of discrete basis functions has evolved as a more general numerical approach to electron-molecule scattering. The purpose of this paper is to illustrate the efficiency of SVP using a plane wave. For this reason, a detailed numerical and theoretical analysis of the method has been carried out (see [2]) and the main results will be presented here. The calculations have been performed for electron scattering in the static field of the hydrogen atom.

\section{The Schwinger Variational Principle}

The total Hamiltonian of the system may be written as

$$
H=H_{o}+V,
$$

where $\mathrm{H}_{o}$ describes the two independent colliding systems and where $\mathrm{V}$ is the interaction potential. Let $\Phi_{i}$ and $\Psi_{f}$ be the initial and final free waves while $\Psi_{i}^{(+)}$and 
$\Psi_{f}^{(-)}$denote the solutions of the Lippmann-Schwinger equations

$$
\begin{aligned}
& \left|\Psi_{i}^{(+)}>=\right| \Phi_{i}>+G_{o}^{(+)} V \mid \Psi_{i}^{(+)}> \\
& \left|\Psi_{f}^{(-)}>=\right| \Phi_{f}>+G_{o}^{(-)} V \mid \Psi_{f}^{(-)}>
\end{aligned}
$$

where

$$
G_{o}^{( \pm)}=\frac{1}{\left(E-H_{o} \pm i \epsilon\right)}
$$

are the two Green operators associated with $\mathrm{H}_{o}$. Then, the differential cross section of this collision is given by

$$
\frac{d \sigma}{d \Omega}=\frac{k_{f}}{k_{i}}|f|^{2}
$$

where $\vec{k}_{i}$ and $\vec{k}_{f}$ are, respectively, the initial and final propagation vectors of magnitude $\mathrm{k}$. The scattering amplitude is such that

$$
\left[f_{i f}\right]=<\Psi_{f}^{(-)}|V| \Phi_{i}>.
$$

Lippmann and Schwinger[1] proposed for the quantity $\left[f_{i f}\right]$ the stationary expression

$$
\left[f_{i f}\right]=<\Psi_{f}^{(-)}|V| \Phi_{i}>+<\Phi_{f}|V| \Psi_{i}^{(+)}>-<\Psi_{f}^{(-)}\left|V-V G_{o}^{(+)} V\right| \Psi^{(+)}>
$$

which is known as the bilinear form of the Schwinger variational principle. Expansion of $\mid \Psi_{i}^{(+)}>$in terms of Slater determinants and the requirement of stability with respect to variation of the coefficients leads to

$$
\left[f_{i f}\right]=\frac{<\Psi_{f}^{(-)}|V| \Phi_{i}><\Phi_{f}|V| \Psi_{i}^{(+)}>}{<\Psi_{f}^{(-)}\left|V-V G_{o}^{(+)} V\right| \Psi^{(+)}>}
$$

We call variational formulation the choice

$$
\begin{aligned}
& \left|\Psi_{i}^{(+)}>=\right| \Phi_{i}>=e^{i \vec{k}_{i}} \\
& \left|\Psi_{f}^{(-)}>=\right| \Phi_{f}>=e^{i \vec{k}_{f}} .
\end{aligned}
$$

Using equations (2.1) and (2.2) in (2.) $\left[f_{i f}\right]$ becomes

$$
[f]_{B}=f_{B}^{(1)}\left[\frac{1}{1-f_{B}^{(2)} / f_{B}^{(1)}}\right],
$$

where

$$
f_{B}^{(1)}=<\Phi_{f}|V| \Phi_{i}>
$$

is the scattering amplitude in the first Born approximation (FBA) and where 


$$
\begin{aligned}
f_{B}^{(2)}= & <\Phi_{f}\left|V G_{o}^{(+)} V\right| \Phi_{i}> \\
& =f_{B}^{(2)}-f_{B}^{(1)}
\end{aligned}
$$

$f_{B}^{(2)}$ being the scattering amplitude in the second Born approximation (SBA).

\section{Results and discussion}

We applied the approach described in the preceding section, equation (2.3) to calculate the elastic cross section for the scattering of eletrons by hydrogen atom. The matrix elements of the potential given in (2.3) are easily calculated using the Dalitz transformation [3]. Although the correct treatment of exchange and of polarization effects is known to be important, we surprisingly found that the present static results are in very good agreement with other results. Our results are also useful to establish benchmarck calculations at the static level of approximation, indispensable in the development of a more complete formalism including exchange and polarization effects. Based on previous experience [2, 4], we do not expect this to be a serious limitation at energies above $100 \mathrm{eV}$.

We have calculated integral elastic cross sections at a number of energies for $\mathrm{H}$ atom. The values obtained for the integral cross sections (using also present FBA and SBA) are compared in Table I.

Table I: Integral cross section $\left(\mathrm{a}_{o}^{2}\right)$ : electron - $\mathbf{H}$

\begin{tabular}{|c|c|c|c|c|}
\hline Energy(eV) & FBA(our result) & SBA(our result) & SVP(our result) & Ref.[5] \\
217.6 & 0.446 & 0.996 & 0.528 & 0.529 \\
340.0 & 0.289 & 0.399 & 0.327 & 0.327 \\
489.6 & 0.201 & 0.272 & 0.224 & 0.223 \\
666.4 & 0.147 & 0.199 & 0.162 & 0.161 \\
\hline
\end{tabular}

Theoretical cross section using static-exchange and multichannel effects with the Padè approximation is included for comparion [5]. We have noted that the SVP is a slightly more rapidly convergent procedure. Since, for electron scattering, the only difference between our SVP and [5] is due to multichannel effects, we may conclude that these effects are not important for $\mathrm{E} \geq 100 \mathrm{eV}$.

\section{Aknowledgments}

J.L.S.Lino acknowledges support from FAPESP and NUPEMAP-UBC, São Paulo. 


\section{References}

[1] P. Levay, and B. Apagyi, J. Phys.B 21, 3741. K. Takatsuka and V. McKoy, Phys. Rev. A, 23, (1981), 2352.

[2] A.R. de Farias J.L.S. Lino, Resumos do XXII CNMAC, (1999), 236.

[3] C. J. Joachin, "Quantum Collisions Theory", North-Holland, 1975.

[4] J.L.S. Lino and M.A.P. Lima, in "XX International Conference on the Physics of Electronic and Atomic Collisions", Vienna-Austria, 1997; J.L.S. Lino and M.A.P. Lima, Braz. J. Phys., 30 (2000), to appear.

[5] C.R. Garibotti and P.A. Massaro, J. Phys. B, 4 (1970), 1270. 